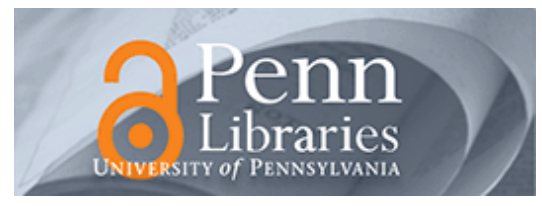

University of Pennsylvania ScholarlyCommons

$1-14-2012$

\title{
Pricing Strategies for User-Provided Connectivity Services
}

\author{
M. H. Afrasiabi \\ University of Pennsylvania
}

Roch A. Guérin

University of Pennsylvania, guerin@acm.org

Follow this and additional works at: https://repository.upenn.edu/ese_papers

Part of the Management Sciences and Quantitative Methods Commons, OS and Networks Commons, and the Systems and Communications Commons

\section{Recommended Citation}

M. H. Afrasiabi and Roch A. Guérin, "Pricing Strategies for User-Provided Connectivity Services", . January 2012.

Afrasiabi, M. H. and Guérin, R. A. "Pricing Strategies for User-Provided Connectivity Services." IEEE INFOCOM 2012 Mini-Conference. Orlando, FL. March 2012.

(C) 2012 IEEE. Personal use of this material is permitted. Permission from IEEE must be obtained for all other uses, in any current or future media, including reprinting/republishing this material for advertising or promotional purposes, creating new collective works, for resale or redistribution to servers or lists, or reuse of any copyrighted component of this work in other works

This paper is posted at ScholarlyCommons. https://repository.upenn.edu/ese_papers/603

For more information, please contact repository@pobox.upenn.edu. 


\title{
Pricing Strategies for User-Provided Connectivity Services
}

\begin{abstract}
User-provided connectivity (UPC) services offer a possible alternative, orcomplement, to existing infrastructure-based connectivity. A userallows other users to occasionally connect through its "home base" inexchange for reciprocation, or possibly compensation. This service modelexhibits strong positive and negative externalities. A large user basemakes the service more attractive, as it offers more connectivity options toroaming users, but it also implies a greater volume of (roaming) trafficpassing through a user's home base, which can increase congestion. Theseinteractions make it difficult to predict the eventual success of such aservice offering, and in particular how to effectively price it. This paperinvestigates a two-price policy where the first price is an introductory price that expires onceservice adoption reaches a certain level. The paper uses a simplifiedanalytical model to investigate pricing strategies under this policy, and their sensitivity to changesin system parameters. The insight and practicalguidelines this yields are validated numerically under morerealistic conditions.

Keywords

pricing, connectivity, user-centric, networks, adoption

Disciplines

Management Sciences and Quantitative Methods | OS and Networks | Systems and Communications

\section{Comments}

Afrasiabi, M. H. and Guérin, R. A. "Pricing Strategies for User-Provided Connectivity Services." IEEE INFOCOM 2012 Mini-Conference. Orlando, FL. March 2012.

(c) 2012 IEEE. Personal use of this material is permitted. Permission from IEEE must be obtained for all other uses, in any current or future media, including reprinting/republishing this material for advertising or promotional purposes, creating new collective works, for resale or redistribution to servers or lists, or reuse of any copyrighted component of this work in other works
\end{abstract}




\title{
Pricing Strategies for User-Provided Connectivity Services
}

\author{
M. H. Afrasiabi and R. Guérin \\ Dept. Elec. \& Sys. Eng., U. Pennsylvania \\ afram@seas.upenn.edu, guerin@ee.upenn.edu
}

\begin{abstract}
User-provided connectivity (UPC) services offer a possible alternative, or complement, to existing infrastructurebased connectivity. A user allows other users to occasionally connect through its "home base" in exchange for reciprocation, or possibly compensation. This service model exhibits strong positive and negative externalities. A large user base makes the service more attractive, as it offers more connectivity options to roaming users, but it also implies a greater volume of (roaming) traffic passing through a user's home base, which can increase congestion. These interactions make it difficult to predict the eventual success of such a service offering, and in particular how to effectively price it. This paper investigates a two-price policy where the first price is an introductory price that expires once service adoption reaches a certain level. The paper uses a simplified analytical model to investigate pricing strategies under this policy, and their sensitivity to changes in system parameters. The insight and practical guidelines this yields are validated numerically under more realistic conditions.
\end{abstract}

\section{INTRODUCTION}

User-provided connectivity (UPC) has emerged as a possible "organic" service model for ubiquitous connectivity. The approach was first made popular by FON (www.fon.com) that introduced a community-based Internet access model, where roaming FON users are allowed access to the Internet through the home connection of other FON users ${ }^{1}$. Home access is through a dedicated device (the FONERA) that connects to the Internet over a standard broadband connection, and allows shared Wi-Fi access (for FON subscribers) to that connection. The main value of the service is in offering connectivity beyond a user's home base, and this value grows with service coverage, i.e., the first user derived no additional value beyond standard Internet connectivity, while a user joining a network with over 4 million FON spots worldwide (as of 2011) clearly sees a much higher value. On the flip-side, a large number of FON users also means greater odds of having to share one's home connection. High levels of roaming traffic ${ }^{2}$ may incite some of the more "sedentary" users who joined early (lack of coverage had little impact on them) to leave. If this happens, coverage may decrease enough to affect the service's value to frequently roaming users, who may in turn start leaving.

In [1], we developed a simple model to elucidate adoption patterns for such a service. Our focus in this paper is on pricing

\footnotetext{
The work was supported by NSF grant CNS-0915982.

${ }^{1}$ See also KeyWifi (http://keywifi.com) for a similar service.

${ }^{2}$ The FONERA can control how much access bandwidth is available to other users, but that share must be high enough to deliver acceptable service to roaming users.
}

strategies and how prices should be set to maximize the profit of a UPC service provider. Pricing policies in general may vary and range from non-discriminatory (same price for all users) to various levels of price discrimination, e.g., see [2] for a review. In this paper, we limit ourselves to a basic twoprice policy, where one price is an introductory price that ends once service adoption reaches a certain level. This is an example of a discriminatory policy, where price discrimination is a function of when a user adopts.

The investigation of this paper is initially analytical in nature, and builds on the simple model of [1]. As argued in Section II, in spite of its simplicity the model captures key aspects of a UPC service adoption, and its analysis provides insight into how to price such a service. The applicability of this insight to more realistic settings is validated numerically for various relaxations and generalizations of the model's assumptions. The paper's contributions are not so much the derivation of "optimal" prices. Its simplified model may in fact not match any real system, so that exact prices are of limited practical relevance. Instead, the paper's main contributions are in using the results of the analysis to develop qualitative pricing guidelines for successful UPC service deployments.

The rest of the paper is structured as follows. Section II introduces the model and its parameters. Solution methods and results are presented in Section III, while Section IV tests and demonstrates their robustness. Finally, Section V gives a brief overview of related works, and Section VI summarizes the paper's results and points to possible extensions.

\section{MOdel Formulation}

This section first summarizes the simplified model introduced in [1] and its applicability to UPC services such as FON. The two-price strategy is then presented, together with the corresponding revenue maximization problem.

\section{A. Service Utility Function}

As commonly done [3], service adoption decisions are based on a utility function, with a user adopting when its utility is non-negative. The simple ${ }^{3}$ utility function we use is given in Eq. (1), whose terms we review next.

$$
U(\theta)=\gamma+\theta(2 x-1)-c m-p_{\theta}
$$

\footnotetext{
${ }^{3}$ The utility function of Eq. (1) is simpler than that of [1], where users can be compensated for external traffic using their Internet access.
} 
$\gamma$ corresponds to the intrinsic utility that any user derives from home connectivity. In the case of FON this corresponds to the value of Internet connectivity and services through the FONERA itself. Because our focus is not on exploring how basic Internet connectivity entices users to sign-up for the service, we assume the same $\gamma$ for all users. Differentiation between users is introduced through the parameter $\theta$ that measures a user's propensity to roam and, therefore, seek Internet access when away from home. $\theta$ is taken to be uniformly distributed in $[0,1]$. A FON user with $\theta=0$ never attempts to connect to the Internet through other users' FONERA, while a user with $\theta=1$ is constantly on the road in need of nearby FONERAs to access the Internet. The former user sees little or no benefits from FON's shared connectivity, while the latter heavily depends on it. Conversely, a $\theta=1$ user imposes its entire Internet traffic on other users', while a $\theta=0$ user has no such impact. These two competing factors are reflected in the terms $\theta(2 x-1)$ and $-c m$, respectively.

The term $\theta(2 x-1)$ measures the service utility to roaming users. It depends on how often a user roams, $\theta$, and the service coverage (adoption), $x$, that ranges from 0 to $100 \%$. Note that when coverage is low $(x<1 / 2)$, roaming utility is negative ${ }^{4}$. In other words, until the odds of finding an available FONERA while roaming are high enough, the limited coverage of the service is a deterrent. As more users join, coverage improves and the service's value grows as it becomes easier for roaming users to locate Internet access. This valuation is weighed by a user's own $\theta$ value that specifies how often it takes advantage of it. The function $-\mathrm{cm}$ is a negative, decreasing function of the volume $m$ of roaming traffic that users generate, i.e., traffic that is directed to other users' Internet access, which captures the negative impact this traffic can have on a user's home access. The parameter $c$ is a normalization factor. Note that the volume of roaming traffic, $m$, grows not just as a function of the number $x$ of adopters, but also as a function of who those adopters are, i.e., their $\theta$ values. Finally, $p_{\theta}$ represents the cost of the service to users, which as the notation indicates could vary across users.

The two-price policy and the profit maximization problem are introduced next.

\section{B. Two-Price Policy}

The major differentiation of a UPC service over traditional home-base connectivity is the ability to connect while roaming. Because this added-value is realized only when adoption is high enough, the adoption timing of a UPC service displays a strong correlation with $\theta$, a user's propensity to roam. Specifically, as a UPC service starts off with limited or no coverage, it is initially attractive only to sedentary users that make up the early adopters. As these users join and coverage correspondingly increases, frequently roaming users gradually find the service more appealing and start joining.

\footnotetext{
${ }^{4}$ As discussed in [1], an equivalent formulation uses a roaming utility that is a positive, increasing function of coverage, and instead scales down the valuation of home connectivity by $(1-\theta)$.
}

The consequence of this adoption pattern is that a low introductory price is typically needed to facilitate the initial build-up of a user-base. However, keeping the price low as coverage increases does not allow the service provider to extract the value created by the higher coverage. This can be remedied by allowing the provider to switch to a higher price once coverage is high enough, i.e., introduce time-based price discrimination. Note that implicit in this two-price policy is that a high final adoption level is realized, i.e., late adopters can be charged more only if they derive greater value from the service because of high coverage. This implies an introductory price low enough to not only bootstrap adoption, but also to ensure that if price was kept unchanged adoption would eventually stabilize at a high level. It is only then, that a higher final price can be safely introduced to recoup some of the added value that the high coverage creates.

Specifically, under a two-price policy, the provider has three decision variables: $p_{i}, p_{f}$, and $x_{t h}$, where $p_{i}$ is the introductory price, and $x_{t h}$ is the service adoption level (coverage) at which pricing switches to the final price $p_{f} \geq p_{i}$. The provider's profit $\Pi\left(p_{i}, p_{f}, x_{t h}\right)$ is then of the form

$$
\Pi\left(p_{i}, p_{f}, x_{t h}\right)=\left(p_{i}-e\right) x_{i}+\left(p_{f}-e\right) x_{f},
$$

where $x_{i}$ and $x_{f}$ denote the number of adopters that at equilibrium pay $p_{i}$ and $p_{f}$, respectively, for the service. $e$ is the exogenous per-customer cost of offering the service. Note that $x_{i} \leq x_{t h}$ as some of the initial adopters may ultimately drop out, i.e., because of the increase in roaming traffic at higher adoption levels. The provider seeks to identify $p_{i}^{*}, p_{f}^{*}$ and $x_{t h}^{*}$ such that

$$
\Pi\left(p_{i}^{*}, p_{f}^{*}, x_{t h}^{*}\right)=\max _{p_{i}, p_{f}, x_{t h}}\left\{\Pi\left(p_{i}, p_{f}, x_{t h}\right)\right\} .
$$

The provider's ability to extract the added value associated with higher coverage depends on both $p_{f}$ and $x_{t h}$. The higher $x_{t h}$, the more valuable the service has become when the price is raised. This allows a higher $p_{f}$, but levied on few users. Conversely, lowering $x_{t h}$ ensures that more adopters are charged $p_{f}$, but it limits how much higher than $p_{i}, p_{f}$ can be. In particular, when the price switch occurs, the $\theta$ value of the last user who joined prior to the switch is $\theta_{t h}=x_{t h}$ [1]. The next user considering joining the service has a $\theta$ value of $x_{t h}^{+}$, but will have to pay $p_{f}$. If $p_{f}$ is too high, this user's utility may be negative, which can then stall adoption.

Ensuring that this does not arise can be formulated more formally as a constraint. It imposes that when switching to price $p_{f}$, the user with $\theta=x_{t h}^{+}$must have positive utility under the new price. This leads to the following two conditions (see again Appendix A of [4] for details) that depend on the position of $x_{t h}$ relative to a threshold function of $c$.

$$
p_{f} \leq \begin{cases}\gamma-\frac{1}{8-2 c} & \text { if } x_{t h}<\frac{1}{4-c} \\ (2-c / 2) x_{t h}^{2}-x_{t h}+\gamma & \text { if } x_{t h} \geq \frac{1}{4-c}\end{cases}
$$

The constraints of Eq. (3) are, however, insufficient to ensure that adoption ultimately converges to a high level after switching to $p_{f}$. As demonstrated in [1] for a single price, in 
some instances adoption need not ever converge to a stable equilibrium. This can also arise under a two-price policy even when $p_{f}$ satisfies the constraint of Eq. (3).

Ensuring convergence to a stable equilibrium calls for additional constraints beyond those of Eq. (3). Unfortunately, the complex dynamics of adoption under a two-price policy make the analysis under those constraints challenging. This is because, unlike what Fig. 1 suggests, the final adoption region under a two-price policy need not be "contiguous." In particular, it is possible for the last adopter paying $p_{i}$, i.e., the adopter with $\theta=x_{t h}$, to have a positive utility, while its immediate neighbor, i.e., the user with $\theta=x_{t h}^{+}$, would ultimately (when adoption levels converge) have a negative utility because of the higher price it pays. The utility of users with higher $\theta$ values may, however, remain positive because of the greater roaming benefits they derive. This creates a "gap" in the range of $\theta$ values of adopters, which makes characterizing final adoption challenging.

As a result and in order to preserve some level of analytical tractability, we introduce the added constraint that the twoprice policy achieves equilibrium while maintaining a contiguous (in $\theta$ ) adoption region. Under this additional constraint, which is shown in Appendix A of [4] to have minimal impact on profit, it is possible to identify an analytically tractable sufficient condition that $p_{f}$ and $x_{t h}$ must meet to ensure the feasibility of an equilibrium, and hence the policy. This condition is given in Eq. (4), where $x=x_{i}+x_{f}$ is the equilibrium adoption level (see details in Appendix A of [4]).

$$
p_{f} \leq(2 x-1) x_{t h}+\gamma-c / 2\left(-x^{2}+2 x\right) \text {, }
$$

\section{SOLUTION AND GUIDELINES}

Under the contiguity requirement, final adoption is as shown in Fig. 1. More importantly, final adoption is equal to that of a single-price policy with price $p_{i}$. Consequently and using results from [1], $x$ is given by

$$
x=\min \left[1, \frac{3-c+\sqrt{(3-c)^{2}+2(4-c)\left(\gamma-p_{i}-1\right)}}{4-c}\right] .
$$

Furthermore, given a continuous adoption region, the set $x_{i}$ and $x_{f}$ of users that pay $p_{i}$ and $p_{f}$, respectively, are as illustrated in Fig. 1 and easily seen to satisfy

$$
\begin{aligned}
x_{i} & =x-\left(1-x_{t h}\right) \\
x_{f} & =1-x_{t h} .
\end{aligned}
$$

This can then be used in Eq. (2) to compute profit as a function of $x_{t h}, p_{i}$ and $p_{f}$ (and the exogenous parameters).

The next step involves maximizing this expression subject to the constraints of Eqs. (3) and (4). This is done in two phases. The first phase involves fixing $p_{i}$ and identifying optimal values for $p_{f}$ and $x_{t h}$ given $p_{i}$. In the second phase, $p_{i}$ is varied to identify the overall optimal combination.

The outcome of this investigation is shown in Fig. 2, with details found in Appendix A of [4]. The figure reports as a function of the exogenous parameter $c(x$-axis) and for different $\gamma$ values (different curves in each sub-figure) the optimal values of the three parameters of the policy $\left(p_{i}, p_{f}, x_{t h}\right)$ and the resulting profit. Unsurprisingly, the profit decreases as $\gamma$ decreases and $c$ increases, since both negatively affect the overall service value. The more interesting aspect for our purpose is how changes in $\gamma$ and $c$ affect the parameters of the two-price policy. In spite of what may seem like a complex set of behaviors, relatively clear trends emerge that we can use to build guidelines for an effective two-price policy. In particular, one can identify three broad regions associated with increasing $c$ values (low, medium, high), which give rise to distinct behaviors for selecting an "optimal" two-price policy.

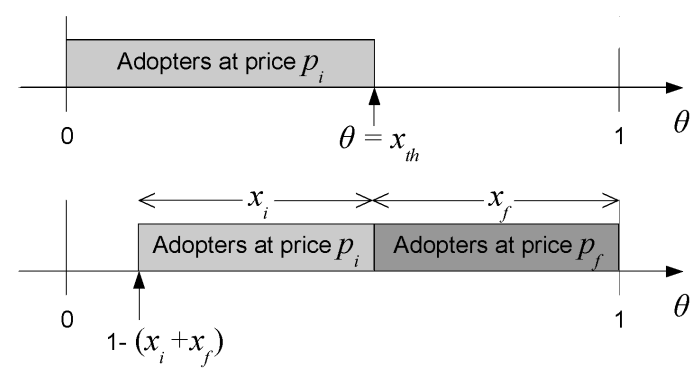

Fig. 1. Adoption under a two-price policy.

In the low $c$ range, i.e., $c \in[0,0.5]$, which is where a UPC service is of most interest, $p_{i}$ and $p_{f}$ follow similar trends (Figs. 2a and 2b). Both prices are relatively insensitive to variations in $c$, and change linearly with $\gamma$ (curves in each figure correspond to differences of 0.1 in $\gamma$, which all produce comparable price drops). Similarly and as long as $c$ remains sufficiently low, the value of $x_{t h}$ (Fig. 2c) is mostly unaffected by changes in either $c$ or $\gamma$. Hence, the structure of the policy that maximizes profit in the low $c$ region is approximately constant: Both $p_{i}$ and $p_{f}$ prices are relatively insensitive to $c$ and decrease roughly linearly with $\gamma$, while the adoption level at which $p_{f}$ is introduced stays fixed at $x_{t h} \approx 75 \%$.

The insensitivity of $x_{t h}$ to both $\gamma$ and $c$ reflects the fact that in the low $c$ region where the impact of roaming traffic is minimal, the service should operate at or near full adoption. This calls for building a reasonably large user-base before switching to the new higher price. The initial build-up of the user base depends on attracting sedentary users who are relatively insensitive to the low coverage. Realizing this is determined primarily by $\gamma$ that represents the bulk of the service value for those users. Hence, the (linear) sensitivity of $p_{i}$ to $\gamma$ is intuitive. The limited sensitivity of $p_{i}$ to $c$ can also be explained by the fact that to build a large enough user base, $p_{i}$ must be low enough to not only attract $\theta \approx 0$ users, but also users with $\theta>0$. The service valuation of those users is negatively affected by the low coverage, and $p_{i}$ must be set low enough to offset that disincentive. This then means that users with $\theta \approx 0$ actually have a positive utility surplus when they join at that price. It is this surplus that allows them to tolerate a (small) increase in $c$ without calling for a corresponding decrease in $p_{i}$. 


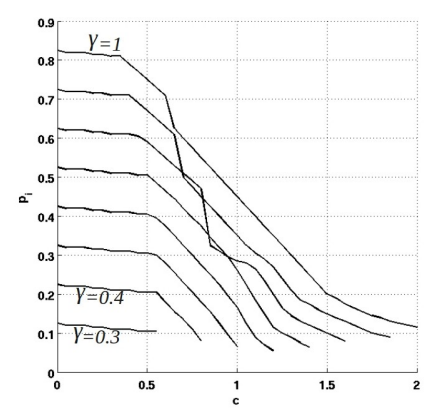

(a) Optimal $p_{i}$

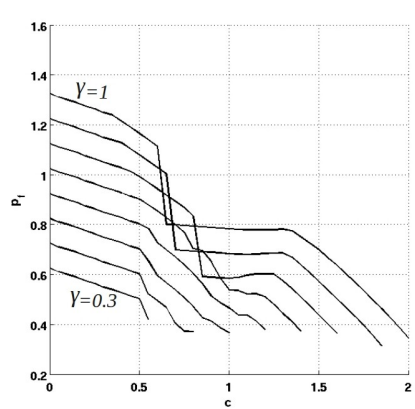

(b) Optimal $p_{f}$

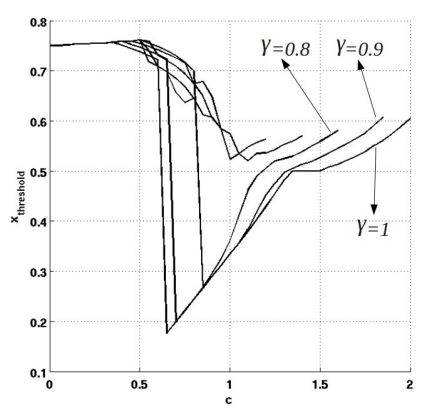

(c) Optimal switching point $x_{t h}$

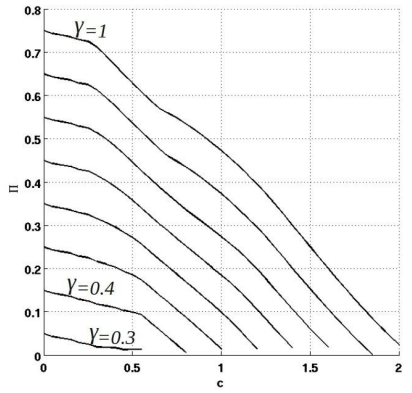

(d) Optimal profit $\Pi$

Fig. 2. Optimal $p_{i}, p_{f}, x_{t h}$ and profit as a function of $\gamma$ at $e=0.2$.

A similar situation arises for $p_{f}$, even if it affects different users, i.e., the $\theta \geq x_{t h}$ users. When adoption crosses $x_{t h}$ and the price switch triggers, Eq. (3) ensures that $p_{f}$ is selected so that the user with $\theta=x_{t h}^{+}$has a positive utility. As adoption increases beyond $x_{t h}$, that user also derives a greater utility from the improved coverage. This factor, even if it is somewhat offset by the negative impact of additional roaming traffic, provides some level of tolerance to increases in $c$. As shown in Fig. 2b, this is not always sufficient to completely avoid some small decrease in $p_{f}$, but it limits its magnitude.

The other two regions that emerge from Fig. 2, are in a range from medium to high $c$ values. Intermediate $c$ values (from $c=0.5$ to about 1.25) see decreases in prices and switching threshold that can exhibit somewhat chaotic behaviors before they settle to some final low level once $c$ is high enough. The $c$ value at which this "settling down" occurs depends itself nearly linearly on $\gamma$, i.e., a higher $\gamma$ corresponds to a larger $c$. This is intuitive since the settling down is associated with configurations where the two-price policy stops being effective, i.e., a high level of adoption is inefficient.

Furthermore, and as illustrated in Appendix A of [4], the chaotic behaviors superimposed on the otherwise relatively steady decline in parameters, can be essentially "ignored" without much impact on the actual profit. Specifically, rather than closely track the fluctuations of the switching threshold $x_{t h}$, it is possible to simply select a threshold value of $x_{t h} \approx 0.5$ that corresponds to the threshold used in the high $c$ region, and similarly ignore fluctuations in either $p_{i}$ and $p_{f}$ and follow the mostly linear decreases that otherwise prevail. The reason why these fluctuations can be ignored has its root in the trade-off between $x_{t h}$ and $p_{f}$. Ignoring a sharp drop in price offsets the effect of ignoring a sharp drop in $x_{t h}$.

The guidelines that result from the above discussion are summarized in Fig. 3 that displays three distinct regions. The region labeled ' $\mathrm{N}$ ' corresponds to configurations where the two-price policy is unable to produce a positive profit. As mentioned earlier, this arises when "forcing" a high level of adoption calls for expensive subsidies (low $p_{i}$ ) to entice enough users to join and stay. These result in negative profits. This happens when $\gamma$ is either very low (irrespective of $c$ ), or too low relative to $c$. For the latter, the cross-over points lie on the boundary between the ' $\mathrm{N}$ ' and ' $\mathrm{B}$ ' regions, which shows a mostly linear relationship between $c$ and $\gamma$. On that boundary, $p_{i}, p_{f}$, and $x_{t h}$ remain essentially constant, with $p_{i} \approx 0$ to attract and keep enough early adopters, $p_{f} \approx 0.3$, and $x_{t h} \approx 0.5$ (under the simple model of Section II-A). In region ' $\mathrm{B}$ ' prices increase nearly linearly when moving towards region 'A' (small $c$ values). When entering 'A', $x_{t h}$ switches to a higher value $\left(x_{t h} \approx 0.75\right)$ and both $p_{i}$ and $p_{f}$ remain unaffected by further decreases in $c$ and increase linearly with $\gamma$. Note that the fact that region 'A' does not extend all the way to $\gamma=0$ is because unless $\gamma \gtrsim e$, a positive profit is not feasible.

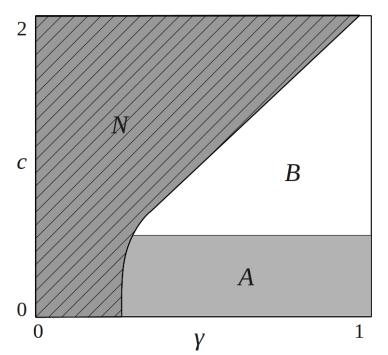

Fig. 3. Pricing guidelines for maximum profit

\section{Robustness Tests}

This section offers numerical evidences of the relative robustness of the guidelines of Section III to errors in the estimation of exogenous parameters and to changes to the model's assumptions, e.g., non-linear externalities and roaming characteristics that follow a non-uniform distribution. Because of space limitations, we report only the results of a sample experiment, with additional results in Appendix B of [4].

Specifically, Fig. 4 displays the cumulative effect of modeling and estimation errors. It assumes that rather than a uniform distribution, $\theta$ follows a bimodal distribution with modes at 0.25 and 0.75 , and that externalities are sub-linear rather than linear, i.e., the users' utility function is of the form

$$
U(\theta)=\gamma+\theta\left(2 x^{0.9}-1\right)-c m^{0.9}-p_{\theta} .
$$


Assuming exogenous parameters with values of $c=0.3, \gamma=$ 0.6 and $e=0.2$, the guidelines of Section III give $p_{i}=0.4$, $p_{f}=0.9$ and $x_{t h}=0.75$. Fig. 4 plots profit (vertical axis), as $c$ and $\gamma$ vary around their assumed values. The thick black dot in the figure marks the profit in the absence of errors, i.e., actual and estimated parameters coincide. The figure shows that while profit eventually degrades as the errors grow large, the policy is robust to a wide range of errors in both $c$ and $\gamma$.

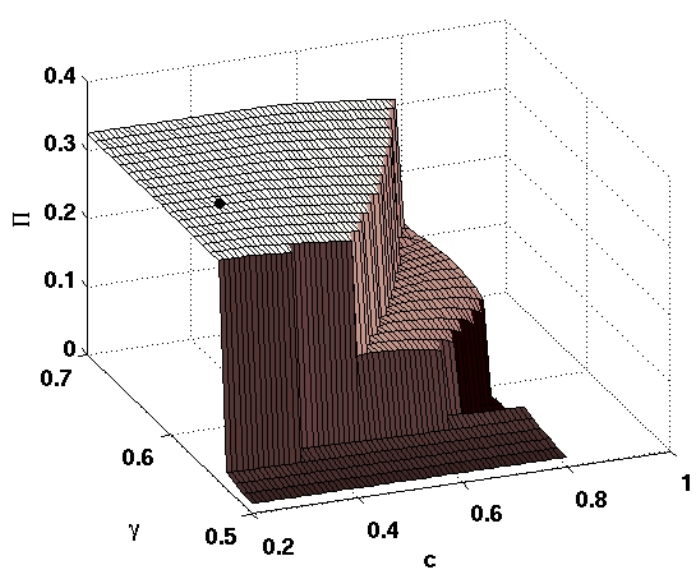

Fig. 4. Robustness of the two-price policy

\section{RELATED WORKS}

There is a vast literature on pricing strategies for products or services that exhibit externalities. It is not our intent to provide an exhaustive review. Instead we focus on a small subset of works most relevant to the problem targeted in this paper.

Early works on pricing in the presence of externalities include [5], [6] that investigated the evolution of prices for products with positive externalities in a monopoly environment. [5] investigated optimal pricing under linear externalities, while [6] was primarily interested in how introductory prices allowed the product to attract a critical mass of adopters.

Optimal pricing in the presence of general positive externalities was more recently investigated in [7] that considered full discriminatory pricing, and showed that an optimal strategy could be computed in polynomial time. Based on the results of its analysis, the paper also proposed what it termed an "influence-and-exploit" strategy that first gives the product away to some users to build critical mass before starting to charge the remaining users. [8] studied mostly the same problem, but under the assumption that prices could only be adjusted at discrete time instants.

The topic of optimal pricing for systems with both positive and negative externalities seems to be have been first addressed in [9] that sought to optimize a combination of provider's profit and consumers' surplus. Different pricing strategies were considered, including flat-rate pricing and pricing strategies that account for the product "amount" consumed by a user".
More recently, [10] considered a similar problem with positive and negative externalities, which arises when introducing a new technology to replace an existing one with which it is compatible. The paper identifies that under the assumption of linear externalities, the optimal pricing strategy is essentially similar to the two-price strategy of Section III.

The main difference between this paper and earlier works is the fact that (negative) externalities depend not just on the number of users that have joined, but also on which users have joined, i.e., the impact of a frequently roaming user differs from that of a sedentary user. The presence of heterogeneity in how users value the service and how they affect its value is a key aspect of a UPC service; one that influences its pricing.

\section{CONCLUSION}

The paper relies on a simple model to develop guidelines for configuring a two-price policy so as to foster the successful deployment of a UPC service. The guidelines are then tested numerically for robustness to various relaxations of the model's simplifying assumptions. The results help identify when a two-price policy that includes a low introductory price can contribute to the eventual success and profit of a UPC service, and conversely when a UPC service is unlikely to succeed irrespective of price.

An extension of interest is to explore service models that include compensation for users that offer access to roaming users. The latter is especially attractive in a context where a Wi-Fi based UPC service is used to offload traffic from a traditional $3 \mathrm{G}$ or $4 \mathrm{G}$ offering, e.g., see [11].

\section{REFERENCES}

[1] M. H. Afrasiabi and R. Guérin, "Exploring user-provided connectivity a simple model," U. Pennsylvania, Technical Report, 2011, available at http://www.seas.upenn.edu/ afram/tr/upc2011.pdf. (A short version was presented at the ICQT 2011 Workshop).

[2] M. Armstrong, "Price discrimination," in Handbook of Antitrust Economics, P. Buccirossi, Ed. MIT Press, 2008.

[3] L. M. B. Cabral, "On the adoption of innovation with network externalities," Mathematical Social Sciences, vol. 19, 1990.

[4] M. H. Afrasiabi and R. Guérin, "Pricing strategies for user-provided connectivity services," U. Pennsylvania, Technical Report, 2011, available at http://www.seas.upenn.edu/ $\sim$ afram/tr/ps2011.pdf.

[5] B. Bensaid and J.-P. Lesne, "Dynamic monopoly pricing with network externalities," International Journal of Industrial Organization, vol. 14, no. 6, pp. 837-855, October 1996.

[6] L. M. B. Cabral, D. Salant, and G. Woroch, "Monopoly pricing with network externalities," International Journal of Industrial Organization, vol. 17, no. 2, pp. 199-214, February 1999.

[7] J. Hartline, V. Mirrokni, and M. Sundararajan, "Optimal marketing strategies over social networks," in Proc. $W W W$, Beijing, China, April 2008.

[8] H. Akhlaghpour, M. Ghodsi, N. Haghpanah, H. Mahini, V. Mirrokni, and A. Nikzad, "Optimal iterative pricing over social networks," in Proc. WINE'10, Stanford U., CA, December 2010.

[9] S. Chao, "Positive and negative externality effects on product pricing and capacity planning," Ph.D. dissertation, Stanford University, 1996.

[10] P. Han, Y. Tan, and J. Peng, "Dynamic pricing for technology upgrades in telecommunication industry," in Proc. ICEC'05, Xi' an, China, August 2005.

[11] D. Burstein, "21st Century triple networks: Ubiquitous 4G, WiFi, \& Wires," CircleID - Internet Infrastucture, January 28, 2011.

\footnotetext{
${ }^{5}$ Our model does not have a notion of product amount consumed by a user but this arises naturally when usage-based pricing is used.
} 